\title{
Anatomia e densidade básica da madeira de Caesalpinia pyramidalis Tul. (Fabaceae), espécie endêmica da caatinga do Nordeste do Brasil ${ }^{1}$
}

\author{
Lazaro Benedito da Silva ${ }^{2,5}$, Francisco de Assis Ribeiro dos Santos ${ }^{3}$, Peter Gasson ${ }^{4}$ e David Cutler ${ }^{4}$
}

\author{
Recebido em 14/03/2008. Aceito em 27/08/2008
}

\begin{abstract}
RESUMO - (Anatomia e densidade básica da madeira de Caesalpinia pyramidalis Tul. (Fabaceae), espécie endêmica da caatinga do Nordeste do Brasil). Este trabalho objetivou estudar a anatomia e a densidade básica da madeira de Caesalpinia pyramidalis utilizada pelas comunidades locais, ocorrente na caatinga de Pernambuco, nos municípios de Serra Talhada e Sertânia, assim como comparar a percentagem dos elementos do lenho no tronco e nos galhos, na própria árvore, a fim de estabelecer o potencial total do lenho para produção de energia. As amostras do lenho do tronco (DAP) e de ramos de seis árvores da espécie foram coletadas nos dois municípios acima citados, em 2002. As análises das amostras seguiram os métodos usualmente empregados em estudo de anatomia de madeiras. Pelos parâmetros anatômicos do lenho e a elevada densidade básica $\left(>0,84 \mathrm{~g} / \mathrm{cm}^{3}\right)$, concluiu-se que Caesalpinia pyramidalis revela grande quantidade de celulose e lignina, portanto apresentou perspectivas seguras para produção de álcool combustível e carvão vegetal. Pode-se propor o uso do lenho do tronco e dos galhos como combustível, desde a fase jovem da madeira, pela grande concentração de fibras, menor concentração de vasos e menor quantidade de parênquima, nos espécimes dos dois municípios.
\end{abstract}

Palavras-chaves: anatomia e densidade da madeira, caatinga, Caesalpinia, carvão

\begin{abstract}
Wood anatomy and basic density of Caesalpinia pyramidalis Tul. (Fabaceae), an endemic species of Northeast Brazil). This work aimed to study the anatomy and basic density of Caesalpinia pyramidalis wood, used by local populations in the municipalities of Serra Talhada and Sertânia (Pernambuco), to assess the percentage of wood elements in the trunk and branches in both localities, and in the tree itself, in order to establish total wood potential for energy production. Samples of the trunk (dbh) and of branches from six trees of the species were collected in 2002. Sample analysis followed the usual methods of wood anatomy studies. Based on the anatomical parameters of the trunk and the high basic density $\left(>0.84 \mathrm{~g} / \mathrm{cm}^{3}\right)$, we conclude that Caesalpinia pyramidalis wood contains an enormous amount of cellulose and lignin. Due to these features, this species showed good perspectives for the production of alcohol and charcoal. The trunk and branches can be used for fuel even at the immature stage because of high fiber concentration, low vessel concentration and low amount of parenchyma at this stage in both municipalities.
\end{abstract}

Kew words: anatomy and density wood, caatinga, Caesalpinia, charcoal

\section{Introdução}

A exploração predatória de madeira aumenta continuamente no mundo, urgindo medidas para implementação de programas para a conservação e manejo sustentável dessas espécies. Cerca de $200.000 \mathrm{~km}^{2}$ de matas naturais da caatinga nordestina foram substituídas por culturas agrícolas e/ou pastagens e a madeira das espécies nativas utilizadas como carvão para uso doméstico e industrial (Sampaio 2002). Juntamente a isto, iniciou-se no País a introdução de espécies de rápido crescimento dos gêneros Eucalyptus L'Her e Pinus L. visando à substituição da madeira de espécies nativas. Existe uma gama de pesquisas com essas espécies, incluindo a análise da qualidade de suas madeiras. Entretanto, pesquisas com espécies nativas não têm tido o mesmo crescimento, especialmente as espécies madeireiras de menor porte, como as da caatinga.

Uma grande preocupação que vem sendo colocada como fundamental no setor florestal é a chamada qualidade intrínseca da madeira destinada à fabricação de carvão vegetal. Isso tem gerado estímulos para diferentes estudos, relacionando as propriedades anatômicas, físicas e químicas da madeira e conseqüen-

\footnotetext{
Parte da Tese de Doutorado do primeiro Autor

2 Universidade Federal da Bahia, Instituto de Biologia, Campus Universitário de Ondina, Ondina, 40270-190 Salvador, BA, Brasil

3 Universidade Estadual de Feira de Santana, Programa de Pós-Graduação em Botânica, Av. Universitária s.n., 44031-460 Feira de Santana, BA, Brasil

4 Jodrell Laboratory, Royal Botanic Gardens, Kew, Richmond, Surrey, TW 93 AB, United Kingdom

5 Autor para correspondência: lbsilva2003@yahoo.com.br
} 
temente a melhoria da qualidade da mesma (Brito \& Barichelo 1979). Em geral, a relação das fibras e a qualidade da madeira são os destaques no estudo da madeira, porém, segundo Sárkáni et al. (1957) e Nguyen (1977) outros componentes estruturais das angiospermas podem influenciar grandemente na qualidade da madeira, tais como as dimensões, proporções e distribuição dos vasos, parênquima axial e radial. Prior \& Cutler (1996) desenvolveram estudos anatômicos do lenho, a fím de buscar informações acerca do potencial energético das madeiras de espécies nativas do Zimbabwe, sul da África, onde $90 \%$ da energia consumida pela comunidade rural provêm das mesmas. Eles identificaram espécies com alto poder energético e calorífico. A similaridade do clima e vegetação despertou o interesse em se fazer estudos similares na região da caatinga nordestina o que tem gerado diferentes projetos e pesquisas.

O presente trabalho faz parte do Programa Plantas do Nordeste, Subprograma Botânica Econômica intitulado "Manejo Sustentado da Vegetação Lenhosa da Caatinga, com Ênfase à Produção de Lenha para uso Doméstico das Comunidades do Nordeste do Brasil". Nesse contexto, incluiu-se o estudo de descrição anatômica da madeira de Caesalpinia piramidalis Tul.

A família Fabaceae, anteriormente Leguminosae e subfamília Caesalpinioideae, compreende, cerca de 158 gêneros (Polhill 1994), porém, segundo Schrire et al. (2005), são 171 gêneros com cerca de 2.250 espécies tropicais e subtropicais. Dentre elas a espécie Caesalpinia pyramidalis é uma das espécies de mais ampla dispersão no nordeste semi-árido podendo ser encontrada em diversas associações vegetais. Ocorre nos Estados do Piauí, Ceará, Rio Grande do Norte, Paraíba, Pernambuco, Alagoas, Sergipe e Bahia, sendo considerada endêmica na caatinga, conhecida popularmente como catingueira e utilizada pelas comunidades pernambucanas de Serra Talhada e Sertânia para a obtenção de lenha e carvão.

Apresenta hábito arbóreo de porte médio, sem espinhos, com 4-6 m de altura, podendo atingir até $12 \mathrm{~m}$. A casca das árvores adultas é de cor cinza-claro, às vezes castanho, com manchas de cor amarelo, verde e branco e libera a camada superficial em lâminas pouco alongadas. O chá é utilizado para tratamento de hepatite e anemia. Além disso, a espécie é indicada para a primeira e a segunda fase de recomposição florestal mista de áreas degradadas (Maia 2004). A madeira é branco-amarelada com cerne escuro, muito pesada, com densidade de $0,99 \mathrm{~g} / \mathrm{cm}^{3}$ (madeira seca), contendo grandes quantidades de celulose e lignina, e é usada como lenha, carvão, estacas, mourões, na construção de casas de taipa e pode ser utilizada para produção de álcool combustível e coque metalúrgico. A cinza da madeira tem elevado teor de potássio e é usada para fabricação de sabão (Maia 2004).

Este trabalho objetivou estudar a anatomia e a densidade básica da madeira de Caesalpinia pyramidalis utilizada pelas comunidades locais ocorrente na caatinga de Pernambuco, nos municípios de Serra Talhada e Sertânia; descrever a anatomia e densidade da madeira; comparar a percentagem dos elementos do lenho no tronco e nos galhos nos espécimes dos dois municípios a fim de estabelecer o potencial total do lenho para produção de energia.

\section{Material e métodos}

Áreas de estudo - foram selecionadas duas áreas de caatinga nas Estações Experimentais da Empresa Pernambucana de Pesquisa Agropecuária (IPA-PE), uma em Serra Talhada (Fazenda Saco - 3.200 ha) e a outra em Sertânia (Fazenda Cachoeira - 630 ha), pelo fato das mesmas apresentarem vasta ocorrência de árvores de Caesalpinia pyramidalis; por estes locais não sofrerem intervenção humana durante a execução do trabalho; por estarem próximas de comunidades locais com necessidade de carvão e de lenha ocasionando o uso indiscriminado desta espécie, necessitando portanto de conhecimentos científicos a cerca da mesma para subsidiar um manejo sustentável da vegetação nestas regiões.

Serra Talhada localiza-se na microrregião do Pajeú, a $418 \mathrm{~km}$ da capital (Recife), com latitude de 07 $53^{\prime}$ '57' $\mathrm{S}$ e longitude de $38^{\circ} 18^{\prime} 09^{\prime}$ 'W, e altitude de 500 s.n.m, apresentando solo maciço de gnais superficial, marrom claro, ácido, franco argiloso com algum cascalho. A precipitação varia de $450-750 \mathrm{~mm}$, de março a maio e a temperatura média anual é de $37^{\circ} \mathrm{C}$. Trata-se de extensa área de caatinga semi-densa, madura, pouco perturbada apresentando estrato arbóreo, com algumas árvores de até $12 \mathrm{~m}$, arbustivo e herbáceo bastante desenvolvidos. As amostras foram coletadas na região denominada de Pimenteira (PROBIO 2000).

Sertânia localiza-se na microrregião de Moxotó, a $316 \mathrm{~km}$ da capital, com latitude de $08^{\circ} 04^{\prime} 25^{\prime}$ "S e longitude de $37^{\circ} 12$ ' $24^{\prime \prime} \mathrm{W}$, e altitude de 611 s.n.m, apresentando solo superficial, marrom claro, ácido, franco argiloso com algum cascalho. A precipitação varia de 350-600 mm, de março a maio e a temperatura média anual é de $35^{\circ} \mathrm{C}$. Trata-se de uma área extensa de caatinga, com vegetação mais fechada na encosta, árvores e arbustos maduros, pouco perturbada. As amostras foram coletadas na região conhecida como Manga do Cerecé (PROBIO 2000).

Para o estudo anatômico do lenho foram coletadas amostras do tronco de três árvores ao nível do diâmetro 
a altura do peito $(\mathrm{DAP}=1,30 \mathrm{~m})$ em cada uma das áreas de estudo, sendo portanto seis indivíduos amostrados. De cada espécime foram coletados dois ramos, o de menor diâmetro e o de maior diâmetro.

Foram preparadas lâminas permanentes contendo uma seção transversal e duas longitudinais tangencial e radial do lenho, com 18-25 $\mu \mathrm{m}$ de espessura utilizandose micrótomo de deslize Spencer. As seções do lenho foram clarificadas com hipoclorito de sódio e coradas com azul de alcião e safranina alcoólica (50\%) 1:1, tratadas em série alcoólica de 50 a $100 \%$, a cada $10 \%$ e montadas em euparol (Johansen 1940).

Nas seções transversais do lenho, em raio contíguo ininterrupto, no sentido da medula para casca, foram mensurados os elementos de vaso, fibras e parênquima radial e longitudinal, utilizando-se o programa de análise de imagem KS 300 da Carl Zeiss, no Jodrell Laboratory do Royal Botanic Gardens Kew. Este programa proporcionou mensurar as áreas, em percentagem, ocupada pelos vasos, fibras e parênquimas radial e longitudinal. Cada seção transversal equivaleu a $100 \%$ de área onde foram analisados a percentagem específica de células. Inicialmente foram marcados todos os vasos presentes na seção e calculada a área ocupada por estes. Em seguida marcou-se a área ocupada pelos raios, parênquima axial e finalmente fibras. A medida que as células eram marcadas o programa desconsiderava esta região, quando da mensuração das demais. Todos os tipos de célula então foram mensurados. Assim, a proporção de cada tipo de célula foi calculada, dando um total de $100 \%$ por área.

Na descrição dos parâmetros anatômicos foram aplicadas as normas da IAWA Committee (1989). As lâminas histológicas permanentes foram depositadas na Xiloteca do Instituto de Biologia da UFBA, do laboratório de micromorfologia da UEFS e do Laboratório de Micromorfologia do Jodrell Laboratory, RBG Kew.

Para a análise da ultraestrutura dos elementos de vasos em microscopia eletrônica de varredura (MEV) foram utilizadas amostras de madeiras de $1 \times 1 \mathrm{~cm}$, no sentido longitudinal à altura da região mediana entre $\mathrm{o}$ alburno e o cerne. Estes cortes foram montados nos porta-espécimes com fita adesiva de grafite e posteriormente recobertos com ouro em sputter Balzers SCD 050. As amostras assim preparadas foram analisadas e eletromicrografadas em microscópio eletrônico de varredura LEO 1430VP.

Na determinação da densidade do lenho, utilizou-se amostras de 2,0 $\mathrm{cm} \times 2,0 \mathrm{~cm}$ da região próxima da medula e da região próxima da casca do DAP e de dois galhos. Essa densidade foi determinada pela razão da massa seca do lenho (seco a $105^{\circ} \mathrm{C}$ ) por volume saturado, de acordo com o método descrito por Vital (1984). Para a comparação das médias e análises de correlação, utilizaram-se os testes $t$ de Student e o Spearman Rank Correlation, respectivamente.

\section{Resultados}

Os dados referentes às alturas, diâmetros dos troncos $(1,30)$, diâmetros dos galhos mais finos e mais grossos e o número de registro das mesmas na xiloteca (em fase de implantação) do Instituto de Biologia da Universidade Federal da Bahia dos seis indivíduos amostrados, sendo três em Sertânia e três em Serra Talhada encontram-se na Tab. 1.

O lenho apresenta camadas de crescimento delimitadas por finas linhas de parênquima marginal descontínuas com uma a duas células de largura que tangenciam faixas de parênquima. Vasos solitários (60\%), múltiplos $(25 \%)$ e em arranjos radiais $(15 \%)$ de 2-4 elementos, porosidade difusa (Fig. 1-4). Elementos de vaso com pontoações intervasculares alternas, guarnecidas (Fig. 5-6); placa de perfuração simples. Pontoações raio-vasculares semelhantes às intervasculares em forma e tamanho. Fibras de paredes muito espessas com lume às vezes quase colapsado (Fig. 1-2); pontoações simples oblíquas; presença de fibras gelatinosas. Parênquima axial aliforme e confluente (Fig. 1-2). Raios estratificados, homogêneos, unisseriados e bisseriados 1-2 células de largura com (11-)15,7(-20) $\mu \mathrm{m}$ e 9-10 células de altura com (124,7-)136,4(-147,8) $\mu \mathrm{m}$ (Fig. 7-8). Cristais prismáticos em células do parênquima axial.

Tabela 1. Dados dos DAP $(1,40)$ e galhos (menores diâmetros e maiores diâmetros) dos indivíduos de Caesalpinia pyramidalis Tul. (catingueira) amostrados em Sertânia e Serra Talhada, PE, Brasil (H = altura; G1 = galho com maior diâmetro; G2 = galho com menor diâmetro; $\mathrm{NR}=$ número de registro na xiloteca do Instituto de Biologia da Universidade Federal da Bahia em fase de implantação).

\begin{tabular}{|c|c|c|c|c|c|c|c|c|c|c|}
\hline \multirow[t]{2}{*}{ Indivíduos } & \multicolumn{5}{|c|}{ Sertânia } & \multicolumn{5}{|c|}{ Serra Talhada } \\
\hline & $\mathrm{H}$ & DAP & G1 & $\mathrm{G} 2$ & NR & $\mathrm{H}$ & DAP & G1 & $\mathrm{G} 2$ & NR \\
\hline 01 & 6,0 & 6,2 & 3,2 & 4,0 & 010 & 5,3 & 4,5 & 2,0 & 3,0 & 013 \\
\hline 02 & 5,0 & 6,0 & 3,0 & 3,2 & 011 & 5,6 & 5,9 & 2,5 & 2,8 & 014 \\
\hline 03 & 6,5 & 6,8 & 3,4 & 4,8 & 012 & 5,0 & 5,0 & 2,1 & 3,2 & 015 \\
\hline
\end{tabular}


Tronco - Aplicando-se o teste $t(P<0,05)$ para comparação de médias, não se observou diferença significativa entre as amostras de Sertânia e Serra Talhada em relação à percentagem de vasos. Já em relação às fibras, observou-se diferença extremamente significativa $(P=0,0003)$, conseqüentemente a percentagem de fibras em Serra Talhada foi maior que em Sertânia. Já a percentagem de parênquima, ao contrário do que aconteceu com as fibras, foi maior em Sertânia do que em Serra Talhada. Semelhante às fibras, a percentagem de raios foi maior nas amostras de Serra Talhada que nas de Sertânia (Tab. 2).

$\mathrm{Na}$ análise da variação medula-casca nas seções transversais do lenho do caule, não houve diferença significativa entre os espécimes dos dois municípios quanto à percentagem de vasos, portanto agruparam-se os espécimes desses dois locais e aplicou-se o teste Spearman Rank Correlation observando-se correlação extremamente significativa $(r=0,47$ e $\mathrm{P}=0,0001)$ entre a distância da medula e a percentagem de vasos, ou seja, à medida que se afasta da medula a percentagem de vasos aumenta. Em relação às fibras, como houve diferença significativa entre os municípios aplicou-se o teste nas amostras tanto de Sertânia como de Serra Talhada, observando-se correlações negativas, isto é, quanto mais distante da medula menor a percentagem de fibras. Assim como os vasos, a percentagem de parênquima também tendeu a aumentar à proporção que se distanciava da medula nos indivíduos dos dois municípios. Em relação aos raios, não houve correlação nas amostras de Sertânia, nem nas de Serra Talhada (Tab. 3; Fig. 9, 10)

As médias de densidade foram altas nos troncos tanto próximos da medula como próximos da casca, não havendo diferenças significativas em relação a essas posições, tampouco em relação aos municípios (Tab. 4).
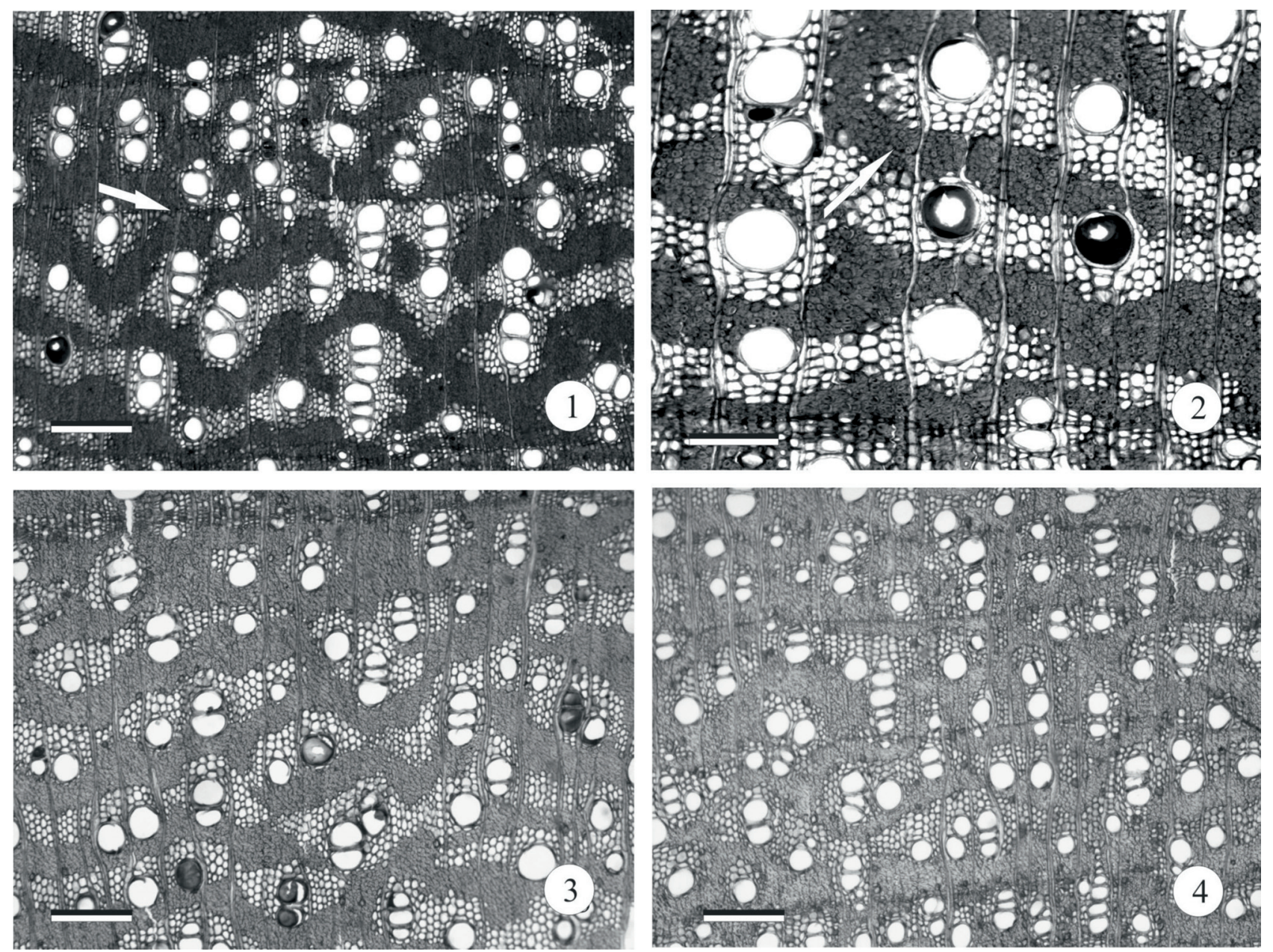

Figuras 1-4. Caesalpinia pyramidalis Tul. (catingueira). Secção transversal. 1. Vasos (poros) de distribuição difusa, solitários, geminados e múltiplos radiais; parênquima marginal em linhas finas (seta) e parênquima aliforme e confluente. 2. Parênquima confluente formando faixas. 3. Galho mais grosso. 4. Galho mais fino. Barras $=200 \mu \mathrm{m}(1,3,4) ; 100 \mu \mathrm{m}(2)$. 
Galhos - Aplicando-se o teste $t$, comparando-se Serra Talhada com Sertânia foram observadas diferenças significativas em relação a proporção de todos os elementos analisados, sendo as maiores percentagens de vasos e parênquima encontradas nas amostras de Serra Talhada e as maiores percentagens de fibras e raios nas amostras de Sertânia (Tab. 2).

Nas amostras de Sertânia as proporções de vasos e parênquima não apresentaram correlação significativa em um dos dois galhos analisados, porém uma tendência à correlação positiva. Todavia, quanto às fibras houve correlação negativa significativa, isto é, quanto mais distante do centro menor a percentagem. Houve, portanto, uma maior concentração de fibras e tendência a menor concentração de vasos e de parênquima no início do crescimento (Tab. 3).

No município de Serra Talhada, os resultados foram semelhantes aos ocorridos em Sertânia. Apenas a percentagem de fibras apresentou correlação negativa com a distância no sentido medula-casca (Tab. 3).

As médias de densidade nos galhos também se mostraram altas, tanto próxima da medula quanto próxima à casca. Assim como ocorreu ao nível do tronco, não houve diferenças significativas em relação a

Tabela 2. Comparação da percentagem média dos elementos do lenho no tronco e nos galhos de Caesalpinia pyramidalis Tul. (catingueira) em Sertânia e Serra Talhada, PE, Brasil, utilizando-se o teste $t$ $(\mathrm{P}<0,05)$.

\begin{tabular}{|c|c|c|c|c|c|c|}
\hline \multirow[t]{2}{*}{ Parâmetros } & \multicolumn{3}{|c|}{ Tronco $(\%)$} & \multicolumn{3}{|c|}{ Galhos (\%) } \\
\hline & Sertânia & & S. Talhada & Sertânia & & S. Talhada \\
\hline Vasos & 18,27 & $=$ & 16,36 & 17,07 & $<$ & 22,02 \\
\hline Fibras & 33,74 & $<$ & 41,46 & 37,24 & $>$ & 29,52 \\
\hline Parênquima & 39,69 & $>$ & 30,39 & 31,92 & $<$ & 36,55 \\
\hline Raios & 8,30 & $<$ & 11,79 & 13,76 & $>$ & 11,91 \\
\hline
\end{tabular}
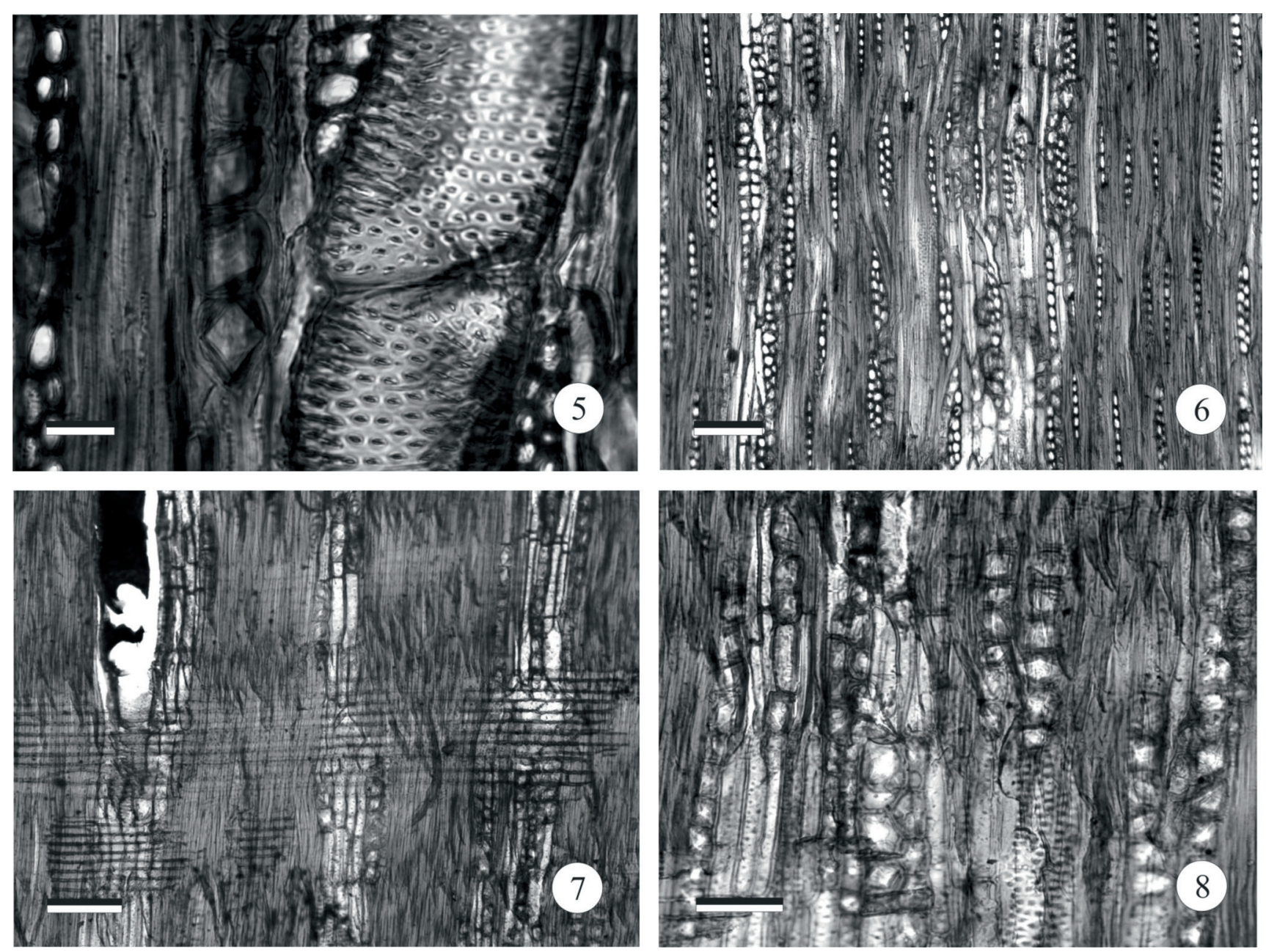

Figuras 5-8. Caesalpinia pyramidalis Tul. (catingueira). 5-7. Secção tangencial. 5. Vaso com pontoações guarnecidas. 6. Raios estratificados, unisseriados e bisseriados. 7. Secção radial. Raio homogêneo. Barras $=20 \mu \mathrm{m}(5) ; 100 \mu \mathrm{m}(6,7) ; 50 \mu \mathrm{m}(8)$. 
essas posições, tampouco em relação aos municípios (Tab. 4).

\section{Discussão}

Os caracteres anatômicos observados em Caesalpinia pyramidalis estão de acordo com aqueles referidos para o gênero por Tortorelli (1956), Latorre (1983), Ranjani \& Krishnamurthy (1991) e Gasson et al. (2003), e para a própria espécie por Paula \& Alves (1980).

A presença de vasos solitários e múltiplos com arranjo difuso é um padrão comum no lenho da maioria das espécies madeireiras. Sendo assim, Caesalpinia pyramidalis inclui-se nesta categoria.

A pontoação guarnecida é uma característica marcante entre as Leguminosas (Jansen et al. 2004). Todos os gêneros das subfamílias Mimosoideae e Papilionoideae apresentam este tipo de pontoação. Apesar de alguns gêneros de Caesalpinoideae não apresentar esta característica, o gênero Caesalpinia L. apresenta este tipo de pontoação. Este caráter pode ser uma informação importante para a filogenia do grupo e para a definição de um largo clado próximo à base da família (Herendeen 2000; Gasson et al. 2003).

Além da pontoação guarnecida ser um traço filogenético, ela pode também representar uma estratégia

Tabela 3. Correlação entre a distância e a percentagem de elementos do lenho da medula para casca, do tronco e galho de Caesalpinia pyramidalis Tul. (catingueira) em Sertânia e Serra Talhada, PE, Brasil, utilizando-se o teste Spearman.

\begin{tabular}{lccc}
\hline Parâmetros & Localidades & Tronco (DAP) & Galhos \\
\hline Vasos & Sertânia & crescente & crescente \\
& Serra Talhada & crescente & crescente \\
Fibras & Sertânia & decrescente & decrescente \\
& Serra Talhada & decrescente & decrescente \\
Parênquima & Sertânia & ns & ns \\
& Serra Talhada & crescente & ns \\
Raios & Sertânia & ns & ns \\
& Serra Talhada & ns & ns \\
\hline
\end{tabular}

ns = não significante

Tabela 4. Variação da densidade básica do lenho no tronco e galhos de Caesalpinia pyramidalis Tul. (catingueira), ocorrente nos municípios de Sertânia e Serra Talhada, PE, localizados no nordeste do Brasil ( $\mathrm{LJ}=$ lenho jovem LA = lenho adulto).

\begin{tabular}{|c|c|c|c|c|c|c|}
\hline & \multicolumn{6}{|c|}{ Densidade básica $\mathrm{g} / \mathrm{cm}^{3}$} \\
\hline & \multicolumn{2}{|c|}{ Tronco } & \multicolumn{2}{|c|}{ Galho 1} & \multicolumn{2}{|c|}{ Galho 2} \\
\hline & $\mathrm{LJ}$ & LA & LJ & LA & LJ & LA \\
\hline Sertänia & 1,01 & 0,98 & 0,96 & 0,93 & 0,95 & 0,95 \\
\hline Serra Talhada & 0,92 & 1,00 & 0,93 & 0,91 & 0,95 & 0,85 \\
\hline
\end{tabular}

adaptativa de sucesso para a sobrevivência e competição de plantas sujeitas a contínuos ou sazonais períodos de seca em regiões de clima quente (Jansen et al. 2004), Assim, Caesalpinia pyramidalis, espécie endêmica da caatinga, apresentou este tipo de pontoação que caracteriza o traço filogenético do gênero e também contribui para a sobrevivência da espécie neste tipo de ecossistema, caracterizado por períodos de seca e com altas temperaturas. As pontoações guarnecidas funcionam como um eficiente sistema hidráulico, prevenindo contra embolias dos vasos (Carlquist 1983, 1988).
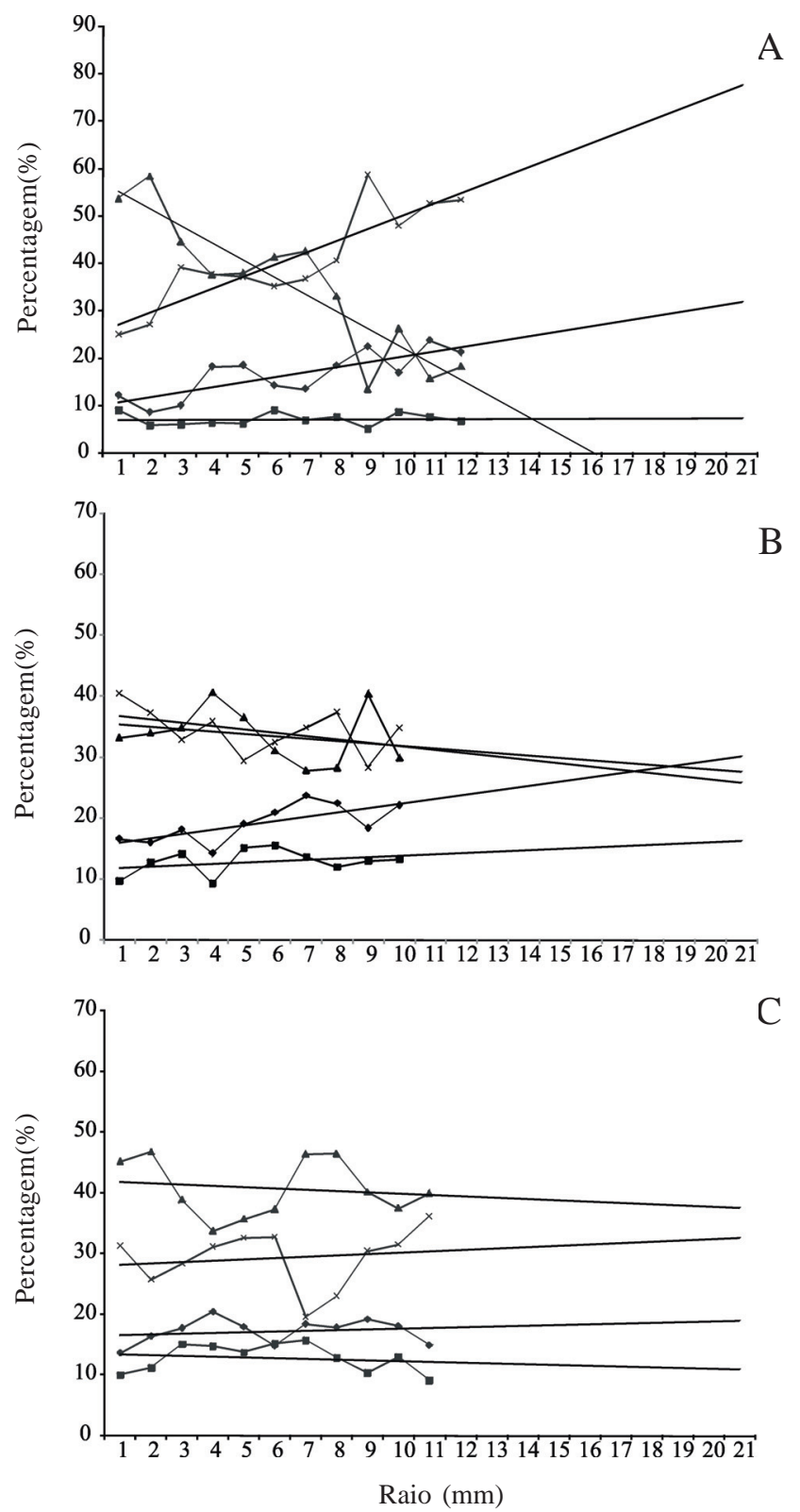

Figura 9. Caesalpinia pyramidalis Tul. (catingueira). Percentagem de vasos, raios, fibras e parênquima axial, no sentido medula casca, de amostras de madeiras coletadas no município de Sertânia, PE, Brasil. A. Tronco ao nível do DAP $(1,30 \mathrm{~m})$. B. Galho 1. C. Galho 2 (-- = Vasos; $-\bullet-=$ Raios; $-\mathbf{-}-=$ Fibras; $-\star-$ = Parênquima $)$. 
Parece existir uma forte correlação negativa entre pontoações guarnecidas e placas de perfuração escalariformes. Em geral, os táxons com pontoações guarnecidas têm placas de perfuração simples. Vasos com pontoações e perfurações destes tipos evitam a embolia em plantas crescendo em ambientes quentes com sazonalidade de estresses hídricos. Este tipo de sistema é mais eficiente quanto à demanda do intenso fluxo de água imposta pelas altas taxas de transpiração em plantas de regiões quentes e secas (Wheeler \& Baas 1991; Jansen et al. 2004).
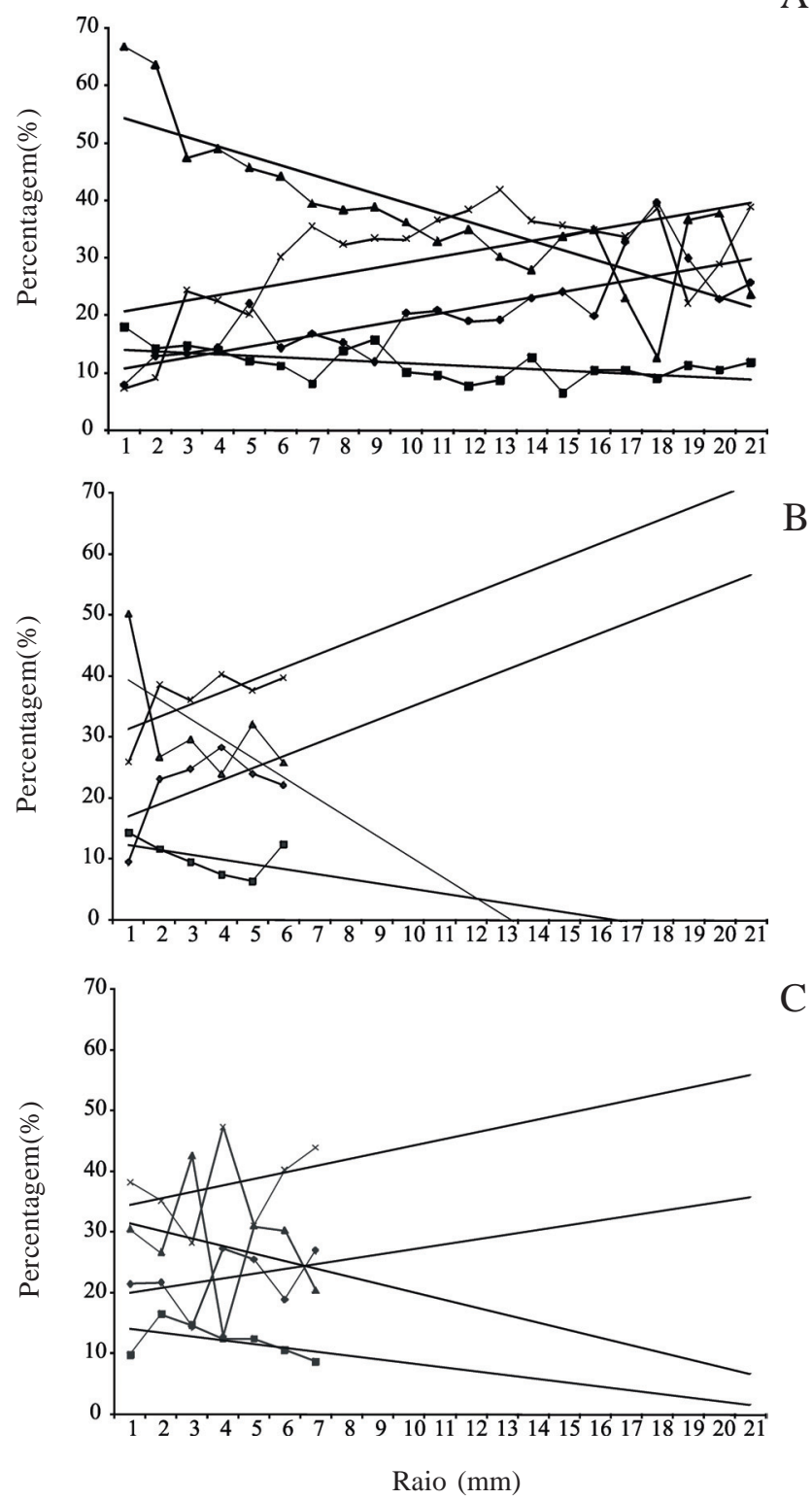

Figura 10. Caesalpinia pyramidalis Tul. (catingueira). Percentagem de vasos, raios, fibras e parênquima axial, no sentido medula de casca de amostras de madeiras coletadas no município de Serra Talhada. A. Tronco ao nível do DAP $(1,30 \mathrm{~m})$. B. Galho 01. C. Galho 02 $\left(-\bullet-=\right.$ Vasos; $-\boldsymbol{-}_{-}^{-}=$Raios; $-\mathbf{-}-=$ Fibras; $-\star-=$ Parênquima).
As fibras gelatinosas podem estar relacionadas ao lenho de tração quando ocorre tortuosidade no caule ou nos galhos, ou terem a função de reserva devido a grande quantidade de água em suas paredes (Paviani 1978). Na espécie em estudo, as fibras gelatinosas parecem estar relacionadas com o armazenamento de água por duas razões: (i) a amostra do lenho coletada não apresentava nenhuma tortuosidade e (ii) pelas características da região da caatinga, onde a baixa precipitação média anual condiciona naturalmente o estabelecimento de uma vegetação caracteristicamente de zona semi-árida, necessitando, portanto, de adaptações para a sobrevivência. Alves \& Angyalossy-Alfonso (2000), analisando a madeira de várias regiões brasileiras, e Angyalossy et al. (2005), analisando Caesalpinia echinata Lam. verificaram essa característica. Neste estudo de Caesalpinia pyramidalis, pode-se também identificar essa característica.

Diversos gêneros da subfamília Caesalpinoideae apresentam uma tendência para parênquima axial aliforme e vasicêntrico, usualmente em bandas, raios estreitos estratificados com até três células de largura e presença de cristais nas células parenquimáticas axiais e radiais e às vezes nas fibras (Gasson et al. 2003). Estes autores afirmaram ainda que estrutura estratificada, composição e tamanho dos raios, presença de cristais nas células radiais e pontoações guarnecidas são caracteres diagnósticos em potencial para análises filogenéticas na subfamília Caesalpinoideae. Assim, os resultados aqui obtidos contribuem com mais dados para estudos taxonômicos e filogenéticos dentro do gênero Caesalpinia.

Baas (1973), Wheeler \& Baas (1991) e Zimmermann (1983) observaram que em ambientes mais quentes a quantidade de parênquima é maior, especialmente em plantas decíduas, como é o caso da espécie aqui estudada da caatinga.

Grisi (1976), citando trabalhos realizados sobre a anatomia de folhas de Caesalpinia pyramidalis, informou que não foram encontrados elementos estruturais que comumente aparecem nos vegetais adaptados à seca, afirmando, portanto, que a importância das características fisiológicas é maior do que as anatômicas na adaptação da planta de caatinga à seca. Concordamos que as características fisiológicas são fundamentais e importantes, porém neste trabalho no qual se demonstra a estrutura do lenho de Caesalpinia pyramidalis, pode-se observar grande concentração de fibras gelatinosas, percentagem considerável de parênquima e distribuição difusa dos vasos, demonstrando que ocorrem variações nos elementos estruturais do lenho que podem estar relacionadas com a questão da seca. 
Além dos fatores climáticos, um outro fato que deve ser levado em consideração é a queda das folhas. No período seco, observa-se que a catingueira perde suas folhas e é uma das primeiras árvores a rebrotar com o início das chuvas. Alvim (1964) observou que a fenologia das árvores mostra relação com a atividade cambial de muitas espécies, sendo esses eventos fenológicos em ciclos anuais ou não anuais. Porém, somente um estudo amplo de dendrocronologia, levando-se em consideração os fatores climáticos e fenológicos, poderá fornecer dados mais precisos sobre essa questão.

Na comparação do lenho dos galhos das duas áreas de estudo, a maior percentagem de fibras ocorreu em Sertânia. Assim, um aspecto importante aqui constatado é que quando a proporção de parede celular das fibras é menos intensa no tronco, como observado nas amostras de Serra Talhada, foi menor nos galhos, como visto em Sertânia e vice-versa. Portanto, ambos os municípios apresentam indivíduos de catingueira com potencial para produção de energia (carvão), e Sertânia pode funcionar como região de manutenção da espécie pelo fato dos galhos apresentarem potencial energético maior.

Aplicando-se o teste de correlação e analisando-se a proporção dos elementos no sentido medula-casca no tronco, observou-se uma maior percentagem de fibras, menor proporção de vasos e menor percentagem de parênquima próximo da medula em Serra Talhada. O mesmo ocorreu em Sertânia. Em casos de utilização desta espécie em manejo sustentável em plantios para produção, provavelmente a mesma poderá ser aproveitada para corte em menor tempo, pois mesmo o lenho na fase jovem se apresenta potencialmente apto para produção de carvão e lenha.

Quando se aplicou o teste de correlação, analisando-se a proporção dos elementos no sentido medula-casca nos galhos, observou-se que houve diferença significativa na percentagem de vasos, fibras e parênquima, semelhante ao ocorrido no tronco e no galho 1 nos dois municípios. Portanto, nesses galhos o lenho apresentou-se apto para produção de carvão e lenha.

Densidade básica - esse é um fator relevante na qualificação da madeira. Segundo Panshin \& De Zeeuw (1980), a densidade é uma propriedade física importante da madeira, pois é um parâmetro que pode afetar outras de suas propriedades. Segundo Chimello (1980), a densidade da madeira é considerada a propriedade física mais importante, relacionando-se com outras propriedades e a sua utilização, assim, madeira de maior densidade corresponde, em geral, a uma maior resistência mecânica e alto valor energético. Isto porque a densidade está diretamente relacionada à quantidade de celulose que a constitui.

Gasson (1987) e Rao et al. (1997) encontraram relação entre densidade do lenho e os vasos. Eles observaram que madeiras com baixa frequiência de vasos apresentaram alta densidade. Fujiwara et al. (1991) observaram em madeiras do Japão, que a espessura da parede das fibras e a percentagem de material da parede têm influência direta na densidade da madeira. Paula (1993) afirmou que quanto mais alta for a massa específica (densidade) da madeira maior será o rendimento de energia em virtude do maior teor de celulose e lignina. Além disso, altas densidades estão relacionadas também com a redução do tamanho das células que compõem a madeira (Angyalossy et al. 2005). Prior \& Gasson (1993) observaram que o comportamento de seis madeiras da África submetidas à carbonização foi influenciado pela natureza e grande percentagem de suas fibras. Portanto, pode-se inferir que Caesalpinia pyramidalis, quando submetida à carbonização produzirá matéria prima de qualidade para obtenção de energia, pois possui uma grande percentagem de fibras e alta densidade em seu lenho.

Como Caesalpinia pyramidalis, aqui estudada, apresentou no alburno e no cerne do tronco e dos galhos, tanto em Serra Talhada quanto em Sertânia, densidade próxima ou maior que $1,0 \mathrm{~g} / \mathrm{cm}^{3}$, podemos afirmar, levando-se em consideração os parâmetros anatômicos analisados anteriormente, que o lenho dessa espécie revela grande quantidade de celulose e lignina, podendo ser utilizado para carvão, álcool combustível e coque metalúrgico.

Inúmeras pesquisas vêm sendo desenvolvidas com o objetivo de atenuar o efeito negativo dos ciclos de curta rotação na qualidade da madeira, através da seleção e do melhoramento genético, aumentando a densidade do lenho juvenil e a uniformidade da densidade ao longo do tronco (Louzada et al. 1994). Os valores de densidade da madeira encontrados no lenho das árvores de Caesalpinia pyramidalis, nas amostras analisadas, próximas da medula e da casca em Sertânia e em Serra Talhada, indicam que desde a fase de formação do lenho juvenil a madeira pode ser aproveitada para fins energéticos. Esse fato torna-se significativo, na atual tendência de utilização da madeira de árvores em ciclos mais curtos, com elevada proporção de lenho juvenil.

Para a geração de energia (carvão) e produção de lenha, o lenho das árvores deve apresentar alto percentual de fibras, baixa proporção de parênquima radial, longitudinal e de vasos e elevada densidade. Estas características físicoanatômicas indicam altos teores de celulose e lignina e, 
em consequiência, a biomassa necessária para a sustentação de uma combustão eficiente. As árvores da espécie estudada mostraram essas características.

Caesalpinia pyramidalis, em Serra Talhada e em Sertânia, apresenta camadas de crescimento que devem estar associadas à pluviosidade e à fenologia, especialmente porque essa espécie é decídua.

Pelos parâmetros anatômicos do lenho aqui analisados (proporção dos vasos, raios, parênquima e proporção e espessura das paredes das fibras) e a elevada densidade básica $\left(0,84-1,01 \mathrm{~g} / \mathrm{cm}^{3}\right)$, conclui-se que Caesalpinia pyramidalis, espécie endêmica da caatinga, revela grande quantidade de celulose e lignina, portanto apresenta potencial para produção de álcool combustível, carvão vegetal e coque metalúrgico.

Pode-se propor o uso do lenho do tronco e dos galhos desde a fase jovem do lenho, devido a grande concentração de fibras, menor concentração de vasos e menor quantidade de parênquima, tanto nos espécimes de Serra Talhada quanto nos de Sertânia.

Em ambos os municípios, Serra Talhada e Sertânia, a Caesalpinia pyramidalis apresentou-se como espécie potencialmente apta para produção de energia, pois quando o seu potencial de celulose e lignina foi menor no tronco no município de Serra Talhada houve uma maior proporção nos galhos e vice-versa. Os galhos apresentam potencial semelhante ao do tronco expresso nas percentagens de fibras, vasos e parênquimas e densidade básica. Pelo potencial dos galhos, reservando as proporções, propõe-se que sejam feitos estudos no sentido de se aproveitar os mesmos e se evitar o corte dos troncos e sacrifício dos indivíduos.

\section{Agradecimentos}

Este trabalho faz parte de um Projeto interinstitutional proposto pela Associação Plantas do Nordeste (APNE), intitulado "Manejo sustentável da vegetação da caatinga com ênfase na produção de carvão para uso doméstico pelas comunidades do Nordeste do Brasil", envolvendo as seguintes instituições: Royal Botanic Gardens, Kew (RBG Kew); Centro Nordestino de Informações sobre Plantas (CNIP); Empresa Pernambucana de Pesquisa Agropecuária (IPA), Universidade Estadual de Feira de Santana (UEFS) e Universidade Federal da Bahia (UFBA), com financiamento do Conselho Nacional de Desenvolvimento Científico e Tecnológico (CNPq), da Comissão de Aperfeiçoamento de Pessoal de Nível Superior (CAPES), do Kew Latin America Research Fellowships Programme (KLARF) e da Clothworkers Guild. Os autores agradecem também a Frans Pareyn e toda a equipe da Associação Plantas do Nordeste
(APNE) pelo apoio total nas coletas do material botânico em campo, Amélia Baracat, Simon Mayo e a equipe do RBG Kew.

\section{Referências bibliográficas}

Alves, E.S. \& Angyalossy-Alfonso, V. 2000. Ecological trends in the wood anatomy of some Brazilian species. IAWA Journal 21: 3-20.

Angyalossy, V.; Amano, E. \& Alves, E.S. 2005. Madeiras utilizadas na fabricação de arcos para instrumentos de corda: aspectos anatômicos. Acta Botanica Brasilica 19: 819-834.

Alvim, P.T. 1964. Periodicidade do crescimento das árvores em climas tropicais. Pp. 405-422. In: Anais do Congresso Nacional de Botânica. 1964. Porto Alegre, Universidade Federal do Rio Grande do Sul.

Baas, P. 1973. The anatomy of Ilex (Aquifoliaceae) and its ecological and phylogenetic significance. Blumea 21: 193-258.

Brito, J.O. \& Barrichelo, L.E.G. 1979. Aspectos florestais e tecnológicos da matéria-prima para carvão vegetal. Circular técnica do Instituto de Pesquisas e Estudos Florestais/ ESALQ-USP 67: 1-20.

Carlquist, S. 1983. Wood anatomy of Onagraceae: further species; root anatomy; significance of vestured pits and allied structures in dicotyledons. Annals of the Missouri Botanical Garden 69: $755-769$.

Carlquist, S. 1988. Comparative wood anatomy: systematic ecological and evolutionary aspects of dicotyledonean wood. Berlin, Springer-Verlag.

Chimelo, J. 1980. Anotações sobre anatomia e identificações de madeiras. São Paulo, IPT.

Fukiwara, S.; Sameshima, K.; Kuroda, K. \& Takamura, N. 1991. Anatomy and properties of Japanese hardwoods I. Variation of fibre dimensions and tissue proportions and their relation to basic density. IAWA Bulletin 12: 419-424.

Gasson, P. 1987. Some implications of anatomical variations in the wood of pedunculate oak (Quercus robur L.) including comparisons with common beech (Fagus sylvatica L.) IAWA Bulletin 8: 149-166.

Gasson, P.; Trafford, C. \& Matthews, B. 2003. Wood anatomy of Caesalpinioideae. Pp. 63-93. In: B.B. Klitgaard \& A. Bruneau (eds.). Advances in Legume Systematics, part 10, Higher Level Systematics. Kew, Royal Botanic Gardens.

Grisi, B.M. 1976. Ecofisiologia da caatinga: comportamento hídrico de Caesalpinia pyramidalis Tul. e Schinopsis brasiliensis Engl. Ciência e Cultura 28: 417-425.

Herendeen, P.S. 2000. Structural evolution in the Caesalpinioideae (Leguminosae). Pp. 45-64. In: P.S. Herendeen \& A. Bruneau (eds.). Advances in Legume Systematics, part 9. Kew, Royal Botanic Gardens.

IAWA Committee.1989. IAWA list of microscopic features for hardwood identification. E.A. Wheeler; P. Baas \& P. Gasson (eds.). IAWA Bulletin 10: 219-332.

Jansen, S.; Baas, P.; Gasson, P.; Lens, F. \& Smets, E. 2004. Variation in xylem structure from tropics to tundra: evidence from vestured pits. Proceedings of the National Academy of Sciences 101: 8833-8837.

Johansen, D.A. 1940. Plant microtechnique. New York, MacGrawHill Book Company.

Latorre, F. 1983. Wood anatomy of eleven legumes from southeast of Ecuador. Ciencia y Naturaleza 10: 84-92. 
Louzada, J.L.P.C.; Fonseca, F.M.A. \& Silva, M.E.C.M. 1994. Relações entre componentes da densidade da madeira no lenho juvenil e no lenho adulto em Pinus pinaster Ait. Pp. 393-405. In: Anais do III Congresso Florestal Nacional. Figueira da Foz, 1994. Figueira da Foz, SFN.

Maia, G.N. 2004. Caatinga, árvores e arbustos e suas utilidades. Fortaleza, Leitura \& Arte.

Marcati, C.R.; Angyalossy-Alfonso, V. \& Benetati, L. 2001. Anatomia comparada do lenho de Copaifera langsdorffii Desf. (Leguminosae-Caesalpinioideae) de floresta e cerradão. Revista Brasileira de Botânica 24: 311-320.

Nguyen, T.V. 1977. Variation in vessel characteristics of Eucalyptus dalrympleana Maiden associated with sample position growth rate and climate changes. Canberra, Australian Nature University.

Panshin, A.J. \& De Zeeuw, C. 1980. Textbook of wood technology. $4^{\text {th }}$ ed. New York, McGraw-Hill Book Co.

Paula, J.E. 1993. Madeiras da caatinga úteis para produção de energia. Pesquisa Agropecuária Brasileira 28: 153-165.

Paula, J.E. \& Alves, J.L.H. 1980. Estudo das estruturas anatômicas e de algumas propriedades físicas da madeira de 14 espécies ocorrentes em áreas de caatinga. Brasil Florestal 10: 47-58.

Paviani, T.I. 1978. Anatomia vegetal e cerrado. Ciência e Cultura 30: $1076-1086$.

Polhill, R.M. 1994. Classification of the Leguminosae. Pp. 45-54. In: F.A. Bisby; J. Buckingham \& J.B. Harbone (eds.). Phytochemistry dictionary of the Leguminosae. New York, Chapman and Hall.

Prior, J.A.B. \& Cutler, D.F. 1996. Radial increments in four tropical, drought tolerant firewood species. Commonwealth Forestry Review 75: 227-233.

Prior, J.A.B. \& Gasson, P.E. 1993. Anatomical changes on charring six african hardwoods. IAWA Journal 14: 77-86.
PROBIO 2000. Seminário sobre avaliação e identificação de ações prioritárias para a conservação, utilização sustentável e repartição de benefícios da biodiversidade do bioma caatinga. Disponível em: http://www.biodiversitas.org/caatinga (Acesso em: 20/07/2001).

Ranjani, K. \& Krishnamurthy, K.V. 1991. Chambered crystal strands in the wood of Caesalpiniaceae. Feddes Repertorium 102: 57-62.

Rao, R.V.; Aebischer, D.P. \& Denne, M.P. 1997. Latewood density in relation to wood fibre diameter, wall thickness, and fibre and vessel percentages in Quercus robur. IAWA Journal 18: 127-138.

Sampaio, E.V.S.B. 2002. Uso das plantas da caatinga. Pp. 49-68. In: V.S.B. Everardo; Sampaio; A.M. Giulietti; J. Virgílio \& C.F.L. Gamarra-Rojas (eds.). Vegetação e flora da caatinga. Recife, APNE \& CNIP.

Sarkani, S.; Stieber, J. \& Fillo, Z. 1957. Investigation on the wood of Hungarian Populus species by means of quantitative xylotomy. Annals University Science Buda (Section Bio) 1: 219-229.

Schrire, B.D.; Lewis, G.P.; Lavin, M. 2005. Biogeography of the Leguminosae. Pp. 21-54. In: G.P. Lewis; B. Schrire; B. Mackinder \& M. Lock (eds.). Legumes of the World. Kew, Royal Botanic Gardens.

Tortorelli, L.A. 1956. Maderas y bosques argentinos. Buenos Aires, ACME, S.A.C.I

Vital, B.R. 1984. Métodos de determinação de densidade da Madeira. Viçosa, UFV.

Wheeler, E.A. \& Baas, P. 1991. A survey of the fossil record for dicotyledonous wood and its significance for evolutionary and ecological wood anatomy. IAWA Bulletin 12: 275-332.

Zimmermann, M. H. 1983. Xylem structure and the ascent of sap. Berlin, Springer Verlag. 